\title{
ESSAI DE CULTURE IN VITRO DE TISSUS DE MOUSTIQUES ET D'INTESTINS DE LAPINS ADULTES INFECTÉS
}

\author{
Par W. GAVRILOV et S. COWEZ
}

En étudiant la méthode de cultures de tissus appliquée à l'étude des protozoaires, nous avons ressenti la nécessité de cultiver des tissus profonds dinsectes.

Ces tissus viscéraux devraient être non seulement stériles au point de vue bactériologique, mais aussi satisfaisants au point de vue protozoologique, c'est-à-dire que, si nous voulons mettre à l'étude, dans une culture de tissu quelconque, une glande de moustique infectée de paludisme, cette glande ne peut pas être porteuse de bacilles, mais doit par contre avoir ses sporozoïtes vivants; ou, si nous prenons les intestins d'un lapin adulte infecté de coccidiose, nous devons avoir ce fragment non seulement dépourvu de bacilles (coli-communis, etc.), mais gardant en outre la possibilité de se reproduire et de conserver les stades de développement des coccidies vivantes. Les moustiques ont habituellement quelques bacilles, ou quelques spores de bacilles ou de champignons sur leur carapace, dans leur œsophage, pharynx et ventricule avec ses sacs accessoires, dans lesquels se conserve le sang prélevé aux animaux. Ce sang se conserve assez longtemps et est un milieu dans lequel vivent différents bacilles, comme les bacilles du foin (Bacillus subtilis), et différents Cocci. Les larves de moustiques nagent dans une eau stagnante pleine de divers bacilles et de Cocci qui adhèrent facilement à leur corps.

En faisant la dissection des moustiques pour extraire des glandes malades, ou saines, des glandes infectées par des protozoaires, ou normales, on travaille toujours dans un milieu plein de bactéries La propreté ou l'habileté avec le:quelles on extrait ces glandes n'ont aucun effet sur leur stérilité, et c'est jourquoi ces glandes ne peuvent être utilisées dans la culture des tissus qui demande une stérilité absolue.

Pour surmonter cette difficulté, nous avons inventé une méthode annales de Parasitologie, T. XVIII, xos 4-5-6. - 1941, p. $180-186$. 
qui nous a donné d'assez bons résultats, et que nous exposons cidessous.

On sait que, grâce à ses petits poils, le moustique est imperméable à l'eau. Si l'on essaie de le désinfecter avec une solution désinfectante, on n'y parvient pas, parce que l'eau ne pénètre pas jusqu'à sa carapace. D'après notre méthode, le moustique, auquel on a enlevé les ailes et les pattes, est aspergé pendant une minute avec de l'alcool méthylique. Ensuite, il est trempé dans une solution de Lugol officinale pendant une ou deux minutes. Après deux minutes de séjour dans cette solution, l'iode et l'iodure de potassium sont enlevés par un lavage prolongé dans une solution d'hyposulfite de soude à 3 p. roo, stérilisé à l'autoclave. Ensuite, cet hyposulfite est éliminé par un lavage à l'eau distillée stérile, ou par une solution de Ringer stérile. Cette manipulation est faite sur un papier filtre stérilisé avec des pipettes Pasteur stériles, munies d'une tétine de caoutchouc.

Après le lavage à l'eau distillée ou à la solution de Ringer, la première étape est terminée. On enlève, avec des instruments stériles, l'abdomen du moustique, on ouvre son thorax et, à còté de la tête, on extrait les glandes salivaires. Cette manipulation est faite sous la loupe binoculaire sur une lamelle de verre stérile. Après extraction, la susdite glande est transportée dans un peu de solution de Ringer stérile sur un porte-objet creux et stérile, et, là, cette glande, qui a été en contact avec le résidu du pharynx, de l'œsophage ou des sacs ventricules ou accessoires pendant l'opération, est lavée avec une solution physiologique ou une solution de Ringer stériles Cette troisième opération est aussi faite avec des pipettes stériles munies d'une tétine en caoutchouc, et, suivant les yeux de l'opérateur, avec ou sans la loupe. Le jet d'eau de la pipette doit être assez fort pour laver les glandes de tous les bacilles qui auraient pu se coller sur elle, et ce lavage, ainsi que l'enlèvement de l'eau de lavage, doivent êtré répétés au moins 6 à ro fois. Il ne faut pas oublier que les glandes des moustiques infectés par le paludisme ont été en contact étroit avec l'œsophage et les sacs accessoires remplis de sang sucé par ce moustique.

Ainsi préparées, les glandes peuvent être considérées comme stériles.

Cette méthode nous a permis de faire la culture de tissus de moustiques, ou la conservation prolongée des organes nécessaires à l'expérimentation. Les larves de moustiques sont traitées de la même manière. L'efficacité de cette méthode nous a été démontrée au cours de nos travaux. Les photographies nous en ont donné les preuves en 
nous permettant de surveiller les battements du cœur des Stegomya fasciata ou Aedes xgypti qu'on voit sur la figure I.

Les organes des moustiques préparés par notre méthode sont placés dans une goutte de plasma de poule additionné de suc embryonnaire de poule et de suc embryonnaire de moustique mème. Ce suc embryonnaire de moustique est préparé par nous en écrasant une grande quantité de larves dans un flacon avec des perles de verre et en les secouant pendant 5 minutes, puis on ajoute quelques centimètres cubes de solution de Ringer, et on filtre sur un filtre Berkefield ou Chamberland L 3 . Ce suc embryonnaire a la propriété de coaguler faiblement le plasma de poule.

Comme on le voit sur la figure 2 (pl. X), les glandes forment jusqu'au $10^{\mathrm{e}}$ jour de petites saillies de cellules qui, souvent, sortent de l'enveloppe de la glande. Sur la figure 4, préparation fixée et colorée au Giemsa, on voit beaucoup de petits points très colorés qui sont des mitoses de cellules de la glande même. La figure 5 nous montre ces mitoses agrandies. Sur la figure 3 , prise sur le vivant, on voit des cellules sortant en saillies et faisant des mitoses.

Jusqu'à présent, il s'agissait d'explantation de glandes d'Anopheles maculipennis, var. atroparvus. Les Stegomya donnent le même résultat. Nous avons réussi à avoir les vraies explantations des cellules de la glande (un peu endommagée) qui sont sorties de son enveloppe et se sont multipliées en faisant une nappe dans laquelle on peut voir le noyau et le protoplasma des cellules en croissance (fig. 3). Les glandes de Stegomya (fixées et colorées sur la figure so), ont une bonne survivance jusqu'au ${ }_{10}{ }^{\circ}$ jour et montrent le noyau des cellules glandulaires et interstitielles. Quand ces glandes commencent à se désagréger, les cellules interstitielles prennent l'aspect de monocytes que nous connaissons des explantations des organes de mammifères. Cé sont vraiment des monocytes, et nous les considérons comme phagocytes (fig. 7). La vie des cellules perdure, et cela nous permet de les utiliser comme milieu pour le développement de protozoaires. Sur la figure 8 , nous voyons les cultures d'un muscle du thorax qui sort de la déchirure de la carapace chitineuse et qui commence à proliférer comme le font habituellement les muscles des mammifères en culture. Sur les cultures jusqu'au $7^{\ominus}$ jour on voit (fig. 9) la même poussée qui a déjà presque pris la forme d'une nappe et qui prolifère plus loin. Sur les figures II et I2, ainsi que I3 et 14 , nous voyons les poussées des fibroblastes, mais il faut noter que ces fibroblastes ne sont pas d'un seul type. La poussée des fibroblastes, très intéressante et très caractéristique des figures $I_{1}$ et I2 $_{2}$, est une poussée de fibroblastes d'un Anophèle. Elle pousse très lentement, on peut cependant distinguer une 
différence entre les figures I 2 et I 3 . Sur la figure I 3 on voit le même noyau dans le prolongement de ces fibroblastes. Les figures $\mathrm{r} 3$ et I 4 montrent les poussées des fibroblastes des Stegomya qui se rapprochent beaucoup des fibroblastes des tissus de mammifères que nous voyons habituellement.

Nous devons noter ici que la température des explantations, c'est-à-dire la température du thermostat, joue un grand rôle, les cultures donnant de meilleurs résultats à $37^{\circ} \mathrm{C}$ jusqu'à 24 ou $25^{\circ} \mathrm{C}$.

Dans sa notice sur les cultures des cellules germinatives et folliculaires, Goldschmidt décrit les résultats obtenus par lui. Il a employé le follicule testiculaire des pupes de Lamia cecropia en hémolymphe. Il ne donne cependant aucun détail sur la méthode d'après laquelle il a prélevé les testicules stérilement, ni comment il a réussi à obtenir de l'hémolymphe, ce qui est pour nous une question essentielle. II a pu voir in vitro la spermatogénèse des nymphes de Lamia cecropia.

D'autres auteurs se sont intéressés à la même question. Les travaux de Goldschmidt ont été confirmés par Lewis et Robertson. La culture de l'épithélium intestinal de la Musca vomitoria et de la Musca domestica a fait l'objet des études de Collier. Mais il est difficile de comprendre comment ses cultures étaient stériles, à moins de supposer que ses mouches, venant d'éclore, n'avaient encore rien sucé.

Quand nous avons essayé d'infecter les cultures de tissus avec des coccidies, nous nous sommes heurtés à l'impossibilité d'obtenir stérilement les oocystes, ou autres formes de coccidies. Sans doute, nous aurions pu tenter d'obtenir des coccidies aseptiques dans les lésions hépatiques dues à Eimeria stiedai, mais nous avons voulu essayer de stériliser avec différents aseptiques le centrifugat des matières fécales d'un lapin infecté de coccidies. N'ayant pu obtenir de résultat satisfaisant, nous sommes revenus à la méthode de lavage de fragments d'intestin dans lequel se sont implantées diverses formes de coccidies.

Pour obtenir stérilement ces fragments habituellement pleins de bacilles coli-communis, streptocoques et, peut-être, autres bactéries, nous avons procédé comme suit. D'un jeune lapin, dans les matières fécales duquel on a trouvé une assez grande quantité d'oocystes d'Eimeria perforans, on coupe un grand morceau (environ $20 \mathrm{~cm}$. de longueur) de l'intestin grêle proche du gros intestin, on l'ajuste à un robinet, et, pendant 5 à ro minutes, avec un très fort jeu d'eau, on enlève les matières fécales et la plus grande quantité des bacilles. Au milieu de ce morceau, on sépare, avec des pinces de Kocher stériles, un fragment duquel on découpe des morceaux plus petits. Le morceau 
central est sectionné longitudinalement et, de sa partie centrale, sont découpés stérilement, avec des scalpels stériles, des carrés de 3 à $4 \mathrm{~cm}^{2}$ que l'on fixe avec des épingles stériles sur un morceau de liège préalablement stérilisé. Avec une pipette Pasteur stérile, munie d'une tétine de caoutchouc, on l'asperge 25 fois de suite à une distance de $5 \mathrm{~cm}$. avec une solution de Ringer stérile. C'est un procédé assez délicat, car il faut non seulement essayer d'asperger la muqueuse, mais aussi les parties péritonéales du morceau d'intestin en question. C'est pourquoi cette aspersion doit être faite avec beaucoup de soin et avec une assez grande force. Avec un morceau d'intestin lavé par cette méthode, on ensemence un bouillon pour vérifier sa stérilité. Ensuite, on découpe $1 \mathrm{~cm}^{2}$ du milieu de ce morceau, qu'on place dans une boìte de Petri stérile. Ce fragment peut servir pour établir directement une culture d'épithélium intestinal parasité de coccidies. Il peut aussi être apposé à une culture déjà établie d'un épithélium embryonnaire indemne de coccidies pour l'infecter.

Ici, j'exposerai seulement les résultats d'explantations d'intestins de lapins adultes infectés de coccidies. Nous avons montré qu'il est possible d'obtenir un morceau d'intestin tout à fait stérile. Mais la poussée épithéliale des intestins du lapin n'est pas facile à obtenir. Les figures I 7 , I8 et I9 nous montrent la poussée de cet épithélium avec les coccidies qui, sur la figure $\mathrm{r} 5$, présentent le stade de développement avancé de trophozoïtes dans les cellules épithéliales. La figure i6 présente l'évolution d'un oocyste dans la culture même, et on voit sur la figure la division en quatre sporocystes La figure 17 montre un oocyste qui, mûri dans l'épithélium même, commence à se diviser en sporoblastes. Mais les figures les plus intéressantes sont les figures 20,21 et 22 . La figure 20 montre l'explantation en nappe de l'épithélium d'intestin du lapin. On voit encore quelques petites inclusions, peut-être des mérozoïtes, dans le plasma de quelques cellules épithéliales. La figure i 9 montre une grande quantité de ces formes trophozoïtes, micro- et macrogamètes dans les diverses cellules d'explantation en nappe. La figure 18 montre un microgamète dans une traînée de tissu épithélial. Ces photographies ont été prises le $12^{\mathrm{e}}$ jour d'explantation, tandis que les photographies non colorées ont été prises les $3^{\mathrm{e}}, 4^{\mathrm{e}}$ et $5^{\mathrm{e}}$ jours d'explantation.

Dans ce travail nous ne comptons pas exposer les résultats obtenus dans l'étude des explantations de coccidies dans la culture des tissus, mais seulement la méthode de stérilisation des organes, ou insectes, qui sont infectés par des bactéries, et dans lesquels nous cherchons à obtenir les protozoaires sans bactéries. Nous pensons que cette méthode peut rendre de réels services dans la protozoologie. 


\section{Bibliographie}

Collier. - Biochemische Feststellung der Verwandtschaft bei Insekten. Z. s. wiss. Insektenbiol., XVI, r920.

Craciun (E. C.). - La culture des tissus en biologie expérimentale. Masson et $\mathrm{C}^{\mathrm{ie}}$, Paris, r93r.

Fauré-FrÉmiet et Ephrussi. - La technique des cultures de tissus in vitro. A. P., I $928,5, \mathbf{I} 57$.

Fischer (A.). - Gewebezüchtung. Handbuch der Biologie der Gewebezellen in vitro, Verlag von Rudolph Müller et Steinicke, München, r93o.

Gavrilov (W.), Bobroff (G.) et Laurencin (M ${ }^{\text {me }}$ S.). - Essai de cultures en tissus de Plasmodium gallinaceum (Brumpt). Ann. Soc. belge méd. trop., XVIII, 1938 .

Gavrilov (W.) et Laurencin ( $\mathrm{M}^{\text {me }} \mathrm{S}$.). - Application d'une méthode de culture de tissus à l'étude des protozoaires. Ann. Soc. belge méd. trop., XVIII, 1938 .

Goldschmid (R.). - Notiz über einige bemerkenswerte Erscheinungen in Gewebekulturen von Insekten. Biol. Zbtrll., XXXVI, I916, p. r6o.

Krontowski (A.) et Rumianzew. - Zur Technik der Gewebskulturen von Kaltblütern in vitro. Pflügers Archiv, CXCX, 1922, p. $29 \mathrm{x}$.

LÉvi (G.). - Explantation, besonders die Struktur und die biologischen Eigenschaften der in vitro gezüchteten Zellen und Gewebe. Zlschr. für die Ges. Anat., XXXI, r934.

Lewis (M. R.) et RoBertson (B.). - The mitochondria and other structures observed by the tissue culture method in the male germ of Chorthippus curtipennis. B.B. W., XXX, ז916, p. 99.

Verne (J.). - La vie cellulaire hors de l'organisme. La cullure des tissus, G. Doin et $\mathrm{C}^{\mathrm{ie}}$, Paris, ${ }^{1937}$.

Instilut d'hygiène de la province d'Anvers et Institut de médecine tropicale Prince-Léopold (Belgique).

Nous exprimons notre reconnaissance à Mlles M.-Th. Van Hoof et G. Goatzen, assistantes à l'Institut de Médecine Tropicale Prince Léopold, Laboratoire du Professeur Rodhain, pour l'aide qu'elles nous ont apportée en nous fournissant des moustiques de diverses espèces et en nous prètant leur concours gracieux. 


\section{EXPLICATION DES PLANCHES X-XII}

\section{Planche $\mathrm{X}$}

Fig. I. - Mitoses des cellules de la glande salivaire d'un Anopheles maculipennis agrandies, colorées au Giemsa.

Fig. 2. - Poussée des cellules de cette glande. On voit les cellules sortir de la glande et se multiplier.

Fig. 3. - Glande de Stegomyia fasciata. L'épithélium de la glande pousse en nappe.

Fig. 4. - Cellules d'une glande salivaire d'Anopheles maculipennis sortant en saillies et se multipliant par mitose.

Fig. 5. - Glande d'un Anophèles maculipennis, fixée et colorée au Giemsa. On voit de nombreux petits points très colorés au Giemsa.

Fig. 6. - Cœur de Stegomyia fasciata, dans du plasma de poule avec suc embryonnaire de larves de moustiques, ayant battu pendant une dizaine de jours.

Fıs. 7. - Glande d'Anopheles maculipennis se changeant en monocytes détachés de la glande après une vingtaine de jours.

Fig. 8. - Culture d'un muscle du thorax, âgée d'une dizaine de jours; Anopheles maculipennis.

Fig. 9. - Même muscle 5 jours après.

\section{Planche XI}

Fig. 1o. - Glande de Stegomyia fasciata fixée et colorée au Giemsa. Survit bien et montre le noyau des cellules glandulaires et interstitielles.

Fig. I1 et 12. - Anopheles maculipennis. Poussée de fibroblastes, culture de Ir à 20 jours.

Fig. 13 et 14 . - Stegomyia. Poussée de fibroblastes qui ressemblent beaucoup aux fibroblastes des mammifères.

\section{Planghe XII}

FIG. I5. - Lapin aduite. Poussée d'intestin de lapin infecté par Eimeria stiedai.

Fıg. r6. - Évolution d'un oocyste dans la culture même. Division en quatre sporocystes.

FIG. 17. - Oocyste, mûri dans l'épithélium même, commençant à se diviser en sporocystes.

Fıg. 18. - Microgamète dans une traînée de tissu épithélial d'intestin de lapin adulte.

Fı́. 19. - Poussée d'épithélium de lapin adulte infecté par des coccidies, fixée et colorée au Giemsa. Divers stades de développement des protozoaires.

Fig. 20. - Explantation en nappe d'épithélium d'intestin de lapin adulte. 

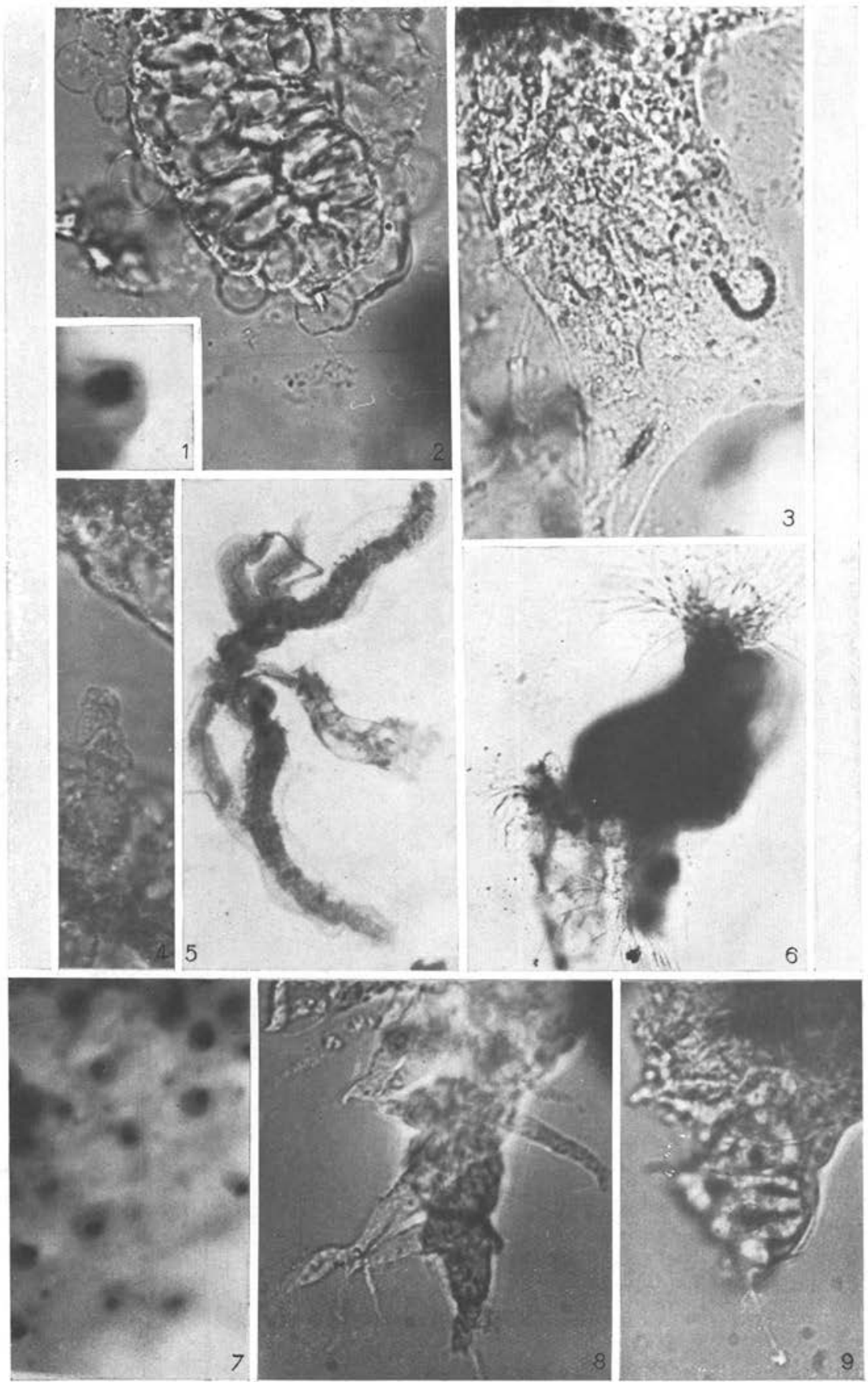

Masson et Cie, Éditeurs 
$\because+1+\frac{2}{6}$ 
ANNALES DE PARASITOLOGIE

T. XVIII, Nos 4-5-6, $194 \mathrm{I}$
Planche XI

(Mémoire Gavrilov)
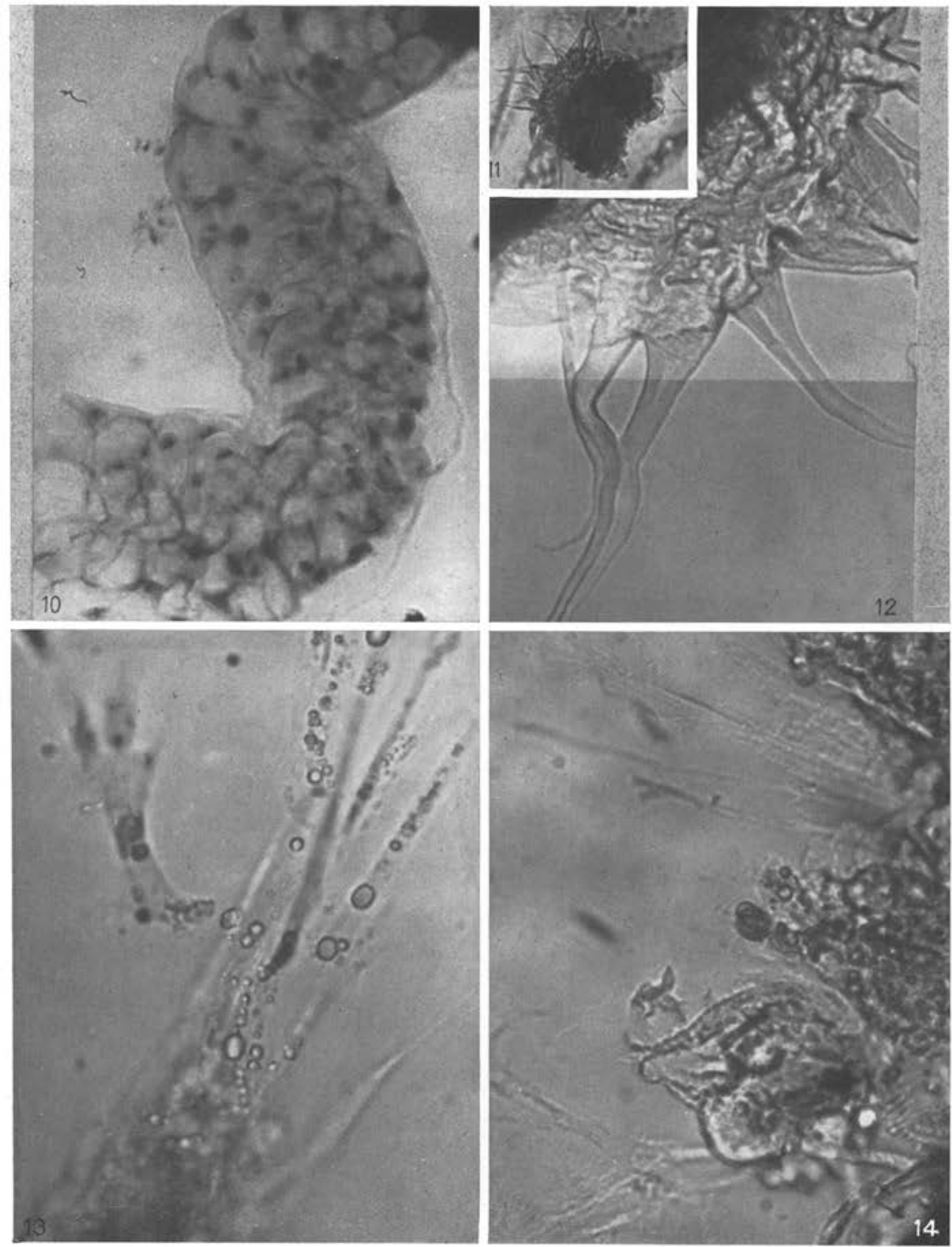

Masson et Cie, Éditeurs 

ANNALES DE PARASITOLOGIE

T. XVIII, Nos 4-5-6, $194 \mathrm{I}$
Planche XII

(Mémoire Gavrilov)
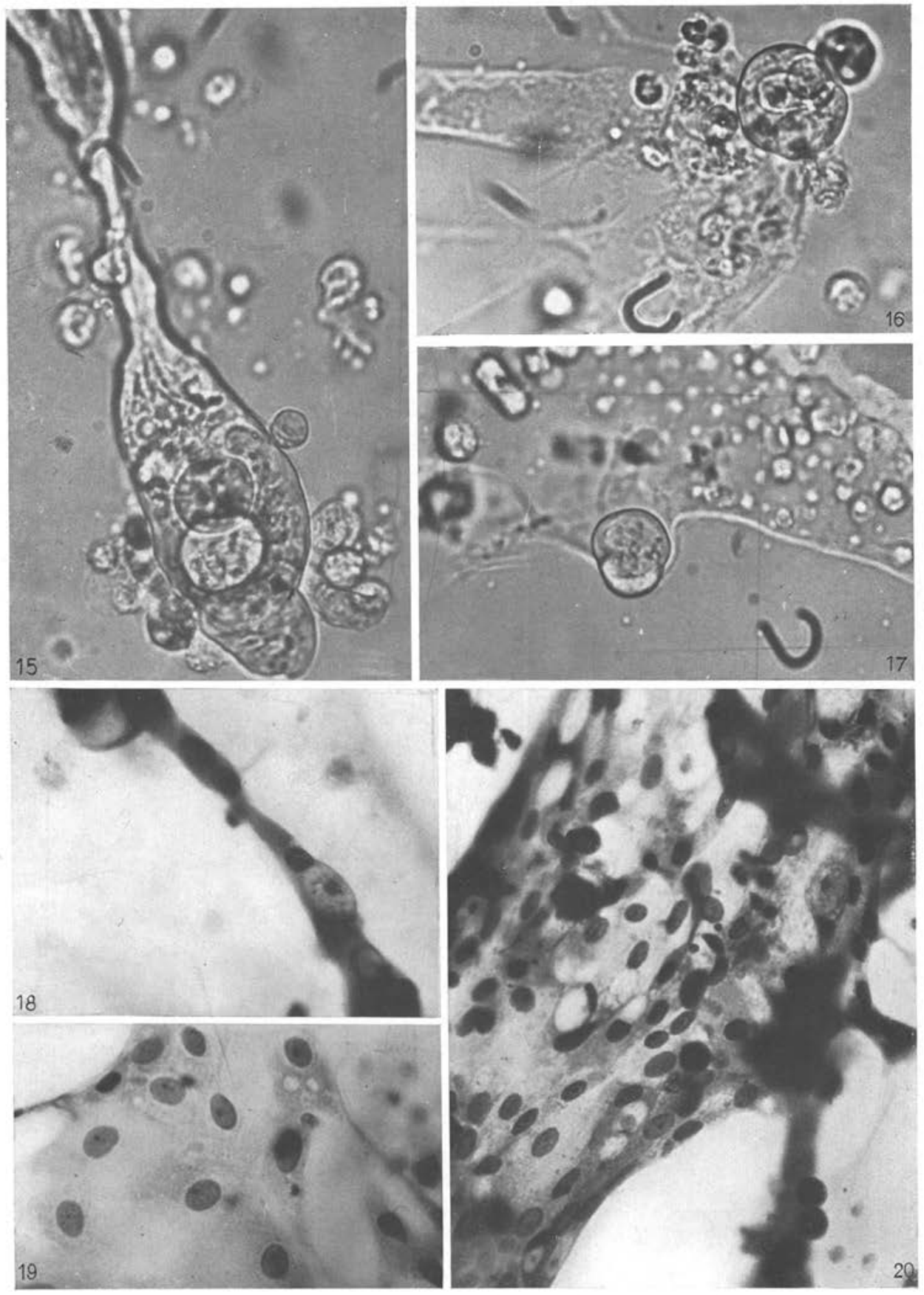

Masson et Cie, Éditeuns 